\title{
Enhanced Cathodoluminescence of KOH-treated InGaN/GaN LEDs with Deep Nano-Hole Amays
}

\author{
Manh-Ha Doan and Jaejin Lee* \\ Department of Electrical and Computer Engineering, Ajou University, Suwon 443-749, Korea
}

(Received January 9, 2014 : April 22, 2014 : accepted May 7, 2014)

\begin{abstract}
Square lattice nano-hole arrays with diameters and periodicities of 200 and $500 \mathrm{~nm}$, respectively, are fabricated on InGaN/GaN blue light emitting diodes (LEDs) using electron-beam lithography and inductively coupled plasma reactive ion etching processes. Cathodoluminescence (CL) investigations show that light emission intensity from the LEDs with the nano-hole arrays is enhanced compared to that from the planar sample. The CL intensity enhancement factor decreases when the nano-holes penetrate into the multiple quantum wells (MQWs) due to the plasma-induced damage and the residues. Wet chemical treatment using $\mathrm{KOH}$ solution is found to be an effective method for light extraction from the nano-patterned LEDs, especially, when the nano-holes penetrate into the MQWs. About 4-fold CL intensity enhancement factor is achieved by the $\mathrm{KOH}$ treatments after the dry etching for the sample with a $250-\mathrm{nm}$ deep nano-hole array.

Keywords: GaN, Light-emitting diode, Nano-hole array, Wet etching, Cathodoluminescence

OCIS codes : (230.3670) Light-emitting diodes; (220.4241) Nanostructure fabrication; (310.3840) Materials and process characterization; (250.1500) Cathodoluminescence
\end{abstract}

\section{INTRODUCTION}

Research and development of GaN-based light emitting diodes (LEDs) have grown rapidly in the last decades due to their promising applications such as back light units for flat panel display, signage, and general lighting [1]. Commercial blue and green LEDs employing $\mathrm{InGaN} / \mathrm{GaN}$ multiple quantum wells (MQWs) are now available [2]. However, there still are a lot of problems that need to be solved in order to achieve the high-efficiency and highbrightness LEDs that are expected to be widely used for general lighting technology instead of conventional light sources such as incandescent and fluorescent bulbs. For example, because of the total internal reflection, only a small fraction of the light generated in the active region of the LED can escape to the surrounding air, resulting in a low external quantum efficiency even if the internal quantum efficiency of the InGaN/GaN LED has been improved substantially by the advanced epitaxial growth techniques [3]. This problem can also be resolved by introducing several techniques such as chip sidewall, surface roughening, patterned substrate, graded-refractive-index coating, and photonic crystal technologies [3,5]. Photonic crystal LED is considered to be a promising approach because it is believed to improve the light extraction efficiency of the LED in two ways. One is the use of the photonic band gaps made by photonic crystal to prevent the guided modes emissions; only light generated in the band gap region can radiate outward since lateral propagation of the guided modes are prohibited in the band gap, thus improving extraction efficiency [5, 6]. The other consists in using photonic crystal to couple the guided modes to radiative modes and diffract those to the air $[7,8]$.

Because of the chemical stability of the GaN material, a dry etching technique such as inductively coupled plasma reactive ion etching (ICP-RIE) is usually employed to fabricate the photonic crystal structures on the $\mathrm{InGaN} / \mathrm{GaN}$ LEDs. Theoretically, the higher light extraction efficiency of the LED is expected from the deeper photonic crystal pattern regardless of the bandgap or the non-bandgap structures [8]. However, the experimental results reveal that light emission intensity is usually reduced when the etchedpatterns penetrate into the MQW active layer due to the plasma-induced damage $[9,10]$. Therefore, most of the studies

\footnotetext{
*Corresponding author: jaejin@ajou.ac.kr

Color versions of one or more of the figures in this paper are available online.
} 
using photonic crystal for the InGaN/GaN LEDs focus on the patterning just on their top p-GaN layer. In this paper, we report on the improvement of cathodoluminescence (CL) of the InGaN/GaN blue LEDs with deep nano-hole arrays, which are penetrated into the MQW regions, fabricated using a combination of the ICP-RIE and wet chemical etching. The dry etching process allows the fabrication of the deep nanopatterns, while the wet chemical etching process is supposed to recover the plasma-induced damage and increase the sidewall areas of the nano-holes, which significantly contribute to the enhancement of the light extraction efficiency of the LED. In addition, we employ a CL mapping technique to investigate the light emission profile from the nano-patterned LEDs. Compared to other optical characterizations such as photoluminescence or electroluminescence, the CL mapping technique has advantages due to the fine probing capability of the electron beam.

\section{EXPERIMENTAL PROCEDURE}

The InGaN/GaN blue LED with a light emission peak at around $430 \mathrm{~nm}$ has been grown by low-pressure metalorganic chemical vapor deposition (LP-MOCVD) on a sapphire (0001) substrate. The LED structure is composed of GaN-based double heterostructures, which includes, from the bottom, a 1- $\mu \mathrm{m}$-thick undoped GaN layer grown on a low-temperature nucleation layer, 4- $\mu$ m-thick Si-doped n-GaN layer, six $\mathrm{In}_{0.3} \mathrm{Ga}_{0.7} \mathrm{~N} / \mathrm{GaN}$ MQWs with well and barrier thickness of 3 and $7 \mathrm{~nm}$, respectively, and a Mg-doped p-GaN top cladding layer of $200 \mathrm{~nm}$. To fabricate the photonic crystal structure, the LED wafer is coated with 300-nm-thick electron beam resist (ZEP-500A). The two dimensional square lattice photonic crystal patterns with circular holes are created by electron beam lithography (JBX 9300FS, JEOL). The diameter and periodicity of the circular holes are intentionally chosen to be 200 and 500 $\mathrm{nm}$, respectively. The samples are then etched by an ICP dry etcher (MULTIPLEX ICP, STS) using a mixture of $\mathrm{BCl}_{3}$ and $\mathrm{Cl}_{2}$ under the ICP source power of $800 \mathrm{~W}$ and the RF bias power of $100 \mathrm{~W}$. Under these conditions, the etching rate for $\mathrm{GaN}$ is measured to be about $5 \mathrm{~nm} / \mathrm{s}$. The ICP etching time is varied to investigate the influences of etching depths on the light emission efficiency of the blue LEDs. After the cleaning process, the samples are divided into two sets. One is used for the CL measurements and the other is transferred to the wet etching process. For the latter, the patterned LEDs are dipped into a 4-M KOH solution at $50^{\circ} \mathrm{C}$ for 20 mins. The InGaN/GaN wafer without a photonic crystal structure, that has undergone the same processes, is used as a reference for the CL measurements. Morphology and etching depth of the photonic crystal structures are investigated by a field-emission scanning electron microscopy (FE-SEM) and a focused ion beam (FIB) technique. The CL measurements are carried out at room temperature using a $10-\mathrm{kV}$ e-beam with a Gatan
MonoCL3 system equipped with a high-sensitivity photo multiplier tube (PMT) attached to an SEM (S-4300SE from Hitachi). With this energy, the electron beam penetrates the GaN layer by about $0.7 \mu \mathrm{m}$ with a quite broad energy deposition profile [11]. There are two ways in obtaining a CL signal from the specimen. One is panchromatic CL images in which the images are formed by plotting integrated luminescence of the wavelength range (160 930 $\mathrm{nm})$ of the PMT detector at each point of the electron beam irradiation. The other is monochromatic CL images that only luminescence at particular wavelength is plotted by adjusting the spectrometer. All the CL images of the samples presented in this study are monochromatic images that are recorded at the wavelength corresponding to the emission peak of the InGaN/GaN MQWs.

\section{RESULTS AND DISCUSSION}

We first study the CL of the patterned LEDs after the ICP etching process with different etching depths. It reveals that all of the patterned LEDs showed a higher CL intensity compared to that of the as-grown LED. The enhancement of the CL intensity is increased with increasing etching depth, achieved a maximum 2-fold higher value at a depth around $150 \mathrm{~nm}$ and then decreased when the etching depth is deeper than this value. This CL enhancement trend of the patterned LED is reasonable because the thickness of the $\mathrm{p}-\mathrm{GaN}$ layer in our $\mathrm{InGaN} / \mathrm{GaN}$ LED is about $200 \mathrm{~nm}$. Higher light extraction efficiency is expected at the deeper etching depth because more guided-modes in the LED structure are expected to have the opportunity to be diffracted to the air [8]. However, when the hole patterns come too near to or penetrate into the MQW layers, the high-energy ICP-RIE process can induce plasma damage effects that can degrade the emission property of the MQW active layer; this may be attributed to the observed CL intensity reduction from the deep-patterned LEDs.

Figure 1 shows the CL mapping images of the LEDs without and with nanohole arrays fabricated by ICP-RIE with etching depths of about 100, 150, and $250 \mathrm{~nm}$. Note that these monochromatic $\mathrm{CL}$ images are recorded at the MQW peak emission wavelength. A non-uniform luminescence profile observed in the planar LED as in Fig. 1(a) may be due to the inhomogeneous In distribution in the InGaN/ GaN MQWs and/or well thickness variation [12]. Interestingly, spatial distribution of the luminescence around the nanoholes is observed to be distinctively different in the two cases. In the case of the etched holes within the p-GaN layer [see Figs. 1(b) and 1(c)], light emission intensity inside the nanoholes is much stronger compared to that at the unpatterned regions as demonstrated by the bright spots in the CL images. When the etching depth penetrates into the MQW layers [see Fig. 1(d)], the light emitted from the MQWs is scattered only at the sidewalls of the nanoholes, and bottom regions of the nanoholes are observed as dark spots in the 


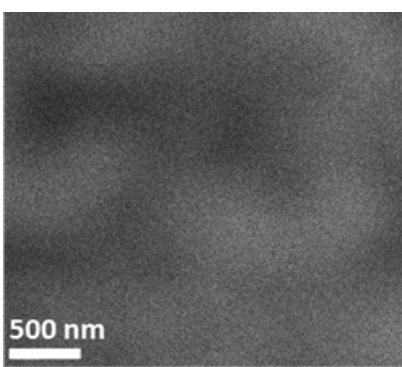

(a)

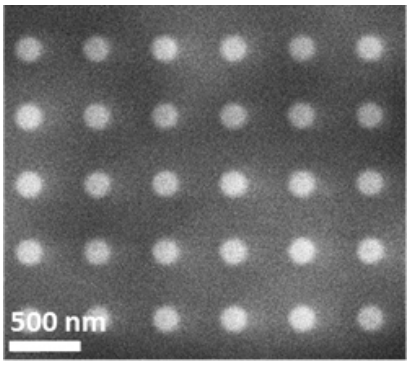

(c)

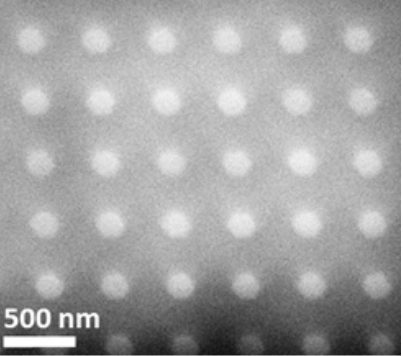

(b)

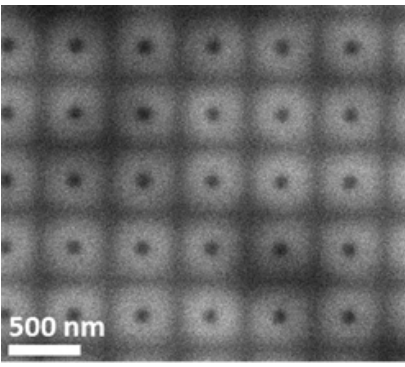

(d)
FIG. 1. Monochromatic CL images of the InGaN/GaN LEDs (a) without nanoholes and with (b) 100-, (c) 150-, and (d) 250-nm-deep nano-hole arrays fabricated by ICP-RIE. The $\mathrm{CL}$ images are recorded at the MQW peak emission wavelength.

CL mapping images. It is worth noting that the soft mask (ZEP-500A) used for the fabrication of the photonic crystal patterns is also slightly etched out during the ICP-RIE process. Therefore, the circular hole patterns tend to become a truncated-inverted-cone (TIC) shape when transferred to the LEDs after relatively long etching time. Experimentally, it is difficult to fabricate a perfect two-dimensional photonic crystal structure with a photonic bandgap for the $\mathrm{InGaN} / \mathrm{GaN}$ blue LED. In addition, it is argued that the enhanced light extraction from the LEDs with the photonic crystal is mainly due to the light scattering by individual nano-holes rather than the photonic crystal band structure [13-14]. In this study, we chose a quite small value of radius/lattice constant ratio of the air holes $(r / a=100 / 500=0.2)$ for our photonic crystal structure. This value is located beyond the region that is expected for a photonic bandgap related with the blue light [15]. By using a small $\mathrm{r} / \mathrm{a}$ value, we may be able to fabricate the TIC shaped deep nanoholes that are quite effective for the light extraction of the $\mathrm{InGaN} / \mathrm{GaN}$ LED due to the enhanced light scattering at the inclinedinner-surface of the etched holes [16-17]. The observed reduction of the CL intensity of the patterned LEDs with the deep nanoholes is mainly caused by plasma-induced damages rather than the removal of active material. It is known that during the dry etching processes of the GaN using ICP-RIE, the surface potential change may induce defects such as nitrogen vacancies and other surface oriented defects on the etched surfaces [18]. These result in a degradation of the electrical and optical characteristics of the InGaN/GaN LED. It is known that the plasma-induced

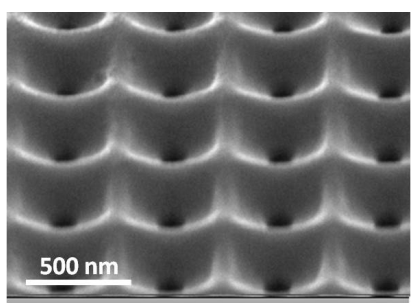

(a)

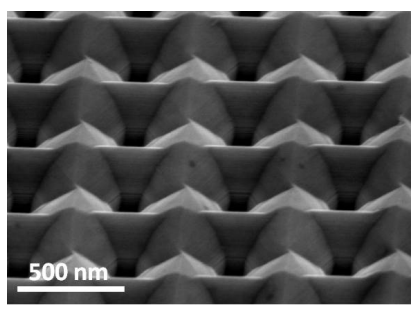

(b)
FIG. 2. SEM images of the 250-nm-deep nano-hole arrays fabricated by ICP-RIE (a) before and (b) after $\mathrm{KOH}$ wet etching.

damages on the surfaces may be recovered by annealing or chemical treatments $[9,10,18]$. In this study, we investigate the effect of $\mathrm{KOH}$ wet chemical treatment on the luminescence properties of the e-beam nano-patterned InGaN/GaN LED, especially when the nano-patterns penetrate into the MQWs.

Figure 2 shows SEM images of the 250-nm deep nanohole arrays fabricated by the ICP etching (a) before and (b) after wet chemical treatment for 20 mins in the $\mathrm{KOH}$ solution. It reveals that, after the wet etching processes, the nanoholes are widened and tend to have an improved hexagonal symmetry. It is worth noting that the hole depth change is observed to be negligibly small, which is consistent with the facts that the $\mathrm{GaN}$ (0001) plane is chemically inert and that the GaN material has anisotropic etching properties, for which the etching rate is quite different for different crystal planes $[19,20]$. To observe inside the deep nano-hole arrays, we carry out cross-sectional SEM measurements using a dual-beam focused ion beam system (FIB-Nova600, Nova Lab). A thin Pt layer is deposited on the sample surface to protect LED epilayers from ion-induced damages and then cross-sectional images are observed by SEM after patterning with an ion beam. Figures 3(a) and 3(b) show cross-sectional SEM images of the 250-nm deep nanoholes before and after $\mathrm{KOH}$ etching, respectively. One can clearly see that inner side walls of the nanoholes are quite rough and the residues appear at their bottom after the ICP etching process [Fig. 3(a)]. After $\mathrm{KOH}$ etching, in contrast, the side walls are smooth and almost no residues are observed inside the nanoholes as can be seen in Fig. 3(b).

Figure 4(a) shows the CL spectra of the LEDs with shallow (150-nm) and deep (250-nm) nanohole arrays before and after $\mathrm{KOH}$ treatments. The blue emission peak of the patterned LEDs is slightly shifted to a shorter wavelength compared to that of the unpatterned sample after the ICP etching process. After $\mathrm{KOH}$ treatment, the emission peaks tend to be recovered to the original position. This may be an additional effectiveness of the $\mathrm{KOH}$ treatment and may need to be investigated in further studies. Figure 4(b) shows the improved CL intensity enhancement factor of the LEDs with nano-hole arrays before and after the $\mathrm{KOH}$ wet etching process. For the calculation of CL intensity enhancement, we took the maximum intensity of the blue emission from 


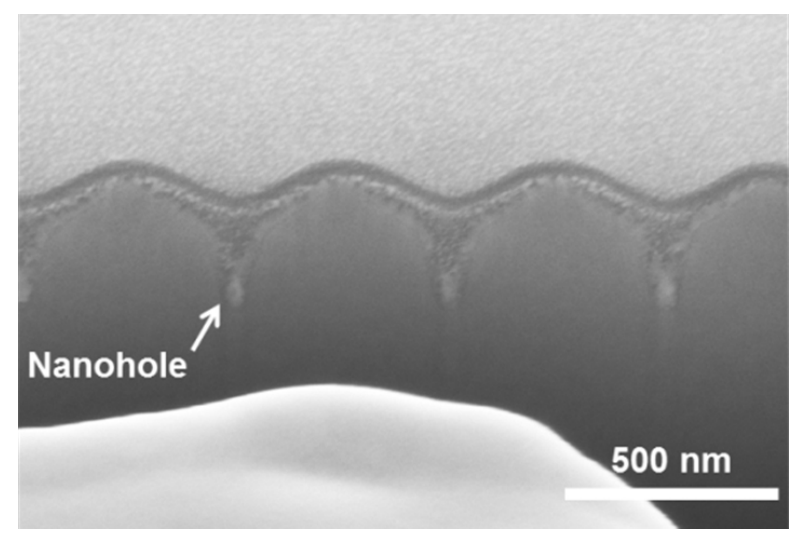

(a)

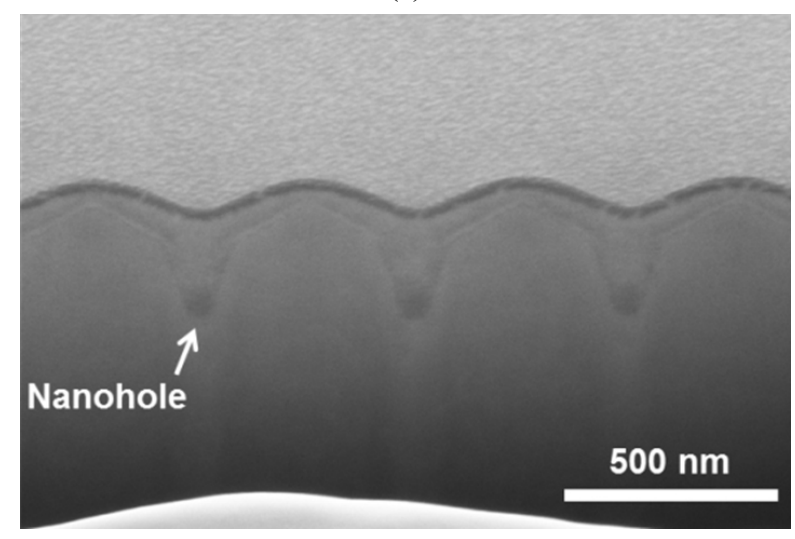

(b)

FIG. 3. Cross-sectional SEM images of the nano-holes (a) before and (b) after $\mathrm{KOH}$ wet etching. The white parts are Pt protection layers deposited during the FIB process. Clean sidewalls of the nano-holes are observed after wet etching as in Fig 3(b).

the CL spectra of each sample. One can see again that the CL intensity of the LEDs with nano-hole arrays fabricated by ICP-RIE is increased with the increasing etching depth, reached a maximum value, and then decreased. All the samples after $\mathrm{KOH}$ wet etching show a higher CL intensity compared to the ones before $\mathrm{KOH}$ wet etching. The enhancement factor, however, is different depending on hole depths; about 4-fold higher enhancement factor is achieved from the nano-hole arrays with the 250-nm deep holes. As we mentioned above, the sidewall formation is effective for the improvement of the light extraction efficiency of the InGaN/GaN LED because it allows more trapped light escape to the air by overcoming the total internal reflection at the surfaces. One can obviously observe that the nanoholes after wet $\mathrm{KOH}$ etching show the better defined sidewalls [see Fig. 2(b)]. Because the depth of the nanoholes is virtually unchanged after the wet $\mathrm{KOH}$ etching, we consider that the improvement of the CL intensity of the nanopatterned LED after $\mathrm{KOH}$ wet etching might originate from the two factors including the recovery of plasma-induced damages and the increased sidewall areas.

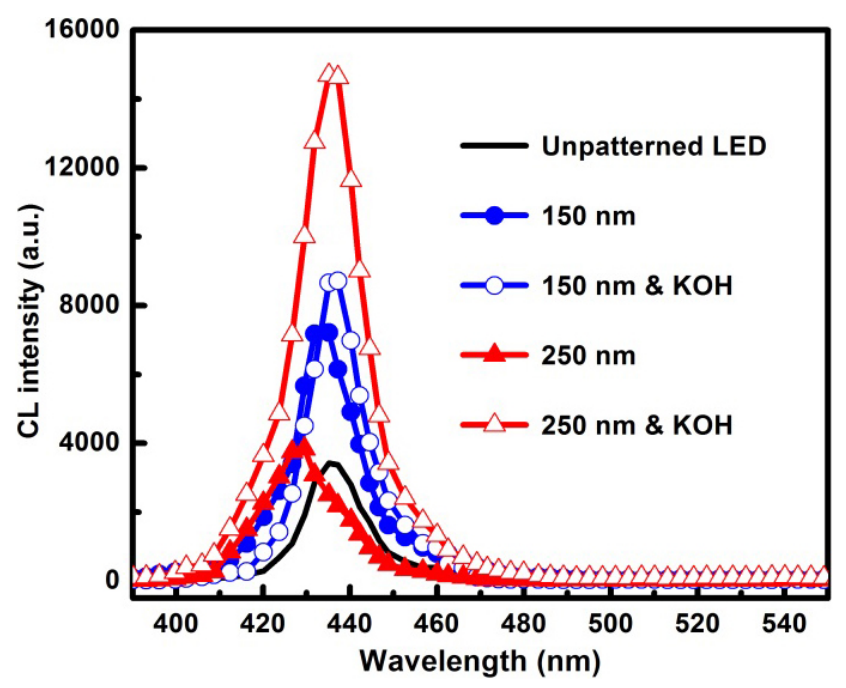

(a)

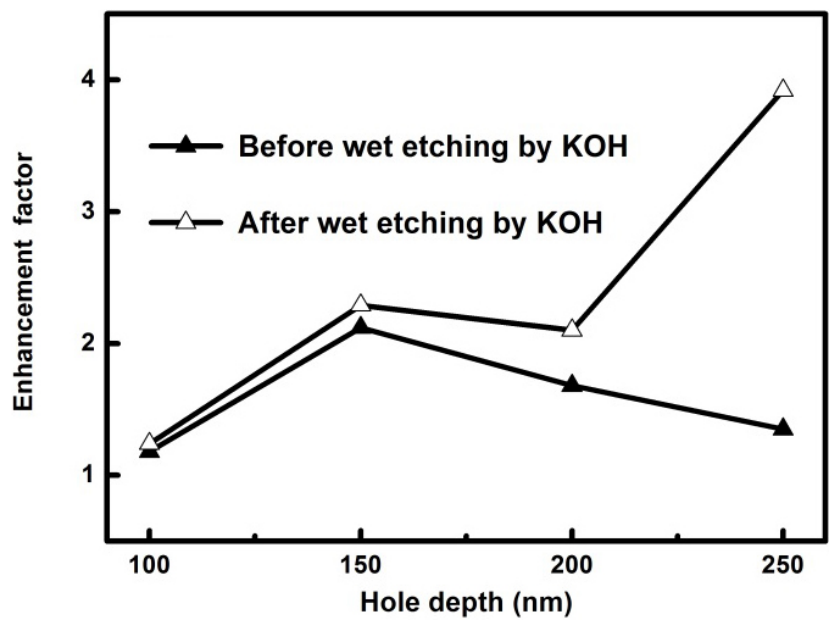

(b)

FIG. 4. (a) CL spectra of the LEDs with shallow (150-nm) and deep (250-nm) nanohole arrays before and after $\mathrm{KOH}$ etching, and (b) CL intensity enhancement factor of the nano-patterned InGaN/GaN LEDs with different nano-hole depths before and after $\mathrm{KOH}$ treatments.

\section{CONCLUSION}

In summary, square lattice nano-hole arrays are fabricated on the InGaN/GaN blue LEDs by the e-beam lithography and ICP-RIE processes. CL investigations show that the light emission efficiency from the LED is enhanced by using the nano-hole patterns. However, the enhancement of CL intensity is decreased when the etched nano-patterns penetrate into the MQW layer due to the plasma-induced damages and the residues. It is found that wet chemical treatment with a $\mathrm{KOH}$ solution followed by the ICP etching significantly improve the CL intensity of the InGaN/GaN LEDs with the nano-hole arrays, especially with the deep nano-hole arrays which deeply penetrate into the InGaN/ GaN MQWs. 


\section{ACKNOWLEDGMENT}

This work is supported by the National Research Foundation Grants (Mid-career Researcher Program: 20090083803).

\section{REFERENCES}

1. E. F. Schubert and J. K. Kim, "Solid-state light sources getting smart," Science 308, 1274-1278 (2005).

2. S. Nakamura, "Current status of GaN-based solid-state lighting," MRS Bulletin 34, 101-107 (2009).

3. P. Niu, Y. Li, X. Li, H. Liu, H. Tian, T. Gao, and G. Yang, "Enhancing the light extraction efficiency of GaN-based LEDs," Proc. SPIE 6828, 682811 (2007).

4. A. N. Noemaun, F. W. Mont, G.-B. Lin, J. Cho, E. F. Schubert, G. B. Kim, C. Sone, and J. K. Kim, "Optically functional surface composed of patterned graded-refractiveindex coatings to enhance light-extraction of GaInN lightemitting diodes," J. Appl. Phys. 110, 054510 (2011).

5. S. Noda, M. Fujita, and T. Asano, "Spontaneous-emission control by photonic crystals and nanocavities," Nat. Photon. 1, 449-458 (2007).

6. J. Y. Kim, M. K. Kwon, K. S. Lee, S. J. Park, S. H. Kim, and K. D. Lee, "Enhanced light extraction from GaN-based green light-emitting diode with photonic crystal," Appl. Phys. Lett. 91, 181109 (2007).

7. J. J. Wierer, M. R. Krames, J. E. Epler, N. F. Gardner, M. G. Craford, J. R. Wendt, J. A. Simmons, and M. M. Sigalas, "InGaN/GaN quantum-well heterostructure light-emitting diodes employing photonic crystal structures," Appl. Phys. Lett. 84, 3885 (2004).

8. A. David, H. Benisty, and C. Weisbuch, "Optimization of light-diffracting photonic-crystals for high extraction efficiency LEDs," IEEE J. Display Technol. 3, 133-148 (2007).

9. J.-M. Lee, C. Huh, D.-J. Kim, and S.-J. Park, "Dry-etch damage and its recovery in $\mathrm{InGaN} / \mathrm{GaN}$ multi-quantum-well light-emitting diodes," Semicond. Sci. Technol. 18, 530-534 (2003).
10. Q. Fan, S. Chevtchenko, X. Ni, S.-J. Cho, F. Yun, and H. Morkoc, "Reactive ion etch damage on GaN and its recovery," J. Vac. Sci. Technol. B 24, 1197-1201 (2006).

11. S. Pereira, M. R. Correia, E. Pereira, K. P. O’Donnell, C. Trager-Cowan, F. Sweeney, and E. Alves, "Compositional pulling effects in InxGa1-xN/GaN layers: A combined depthresolved cathodoluminescence and Rutherford backscattering/ channeling study,” Phys. Rev. B 64, 2053111 (2001).

12. S. Sonderegger, E. Feltin, M. Merano, A. Crottini, J. F. Carlin, R. Sachot, B. Deveaud, N. Grandjean, and J. D. Ganière, "High spatial resolution picosecond cathodoluminescence of InGaN quantum wells," Appl. Phys. Lett. 89, 232109-232111 (2006).

13. D. H. Long, I. K. Hwang, and S. W. Ryu, "Design optimization of photonic crystal structure for improved light extraction of GaN LED," IEEE J. Select. Topics Quantum Electron. 15, 1257-1263 (2009).

14. B. Wang, Y. Jin, and S. He, "Effects of disorder in a photonic crystal on the extraction efficiency of a lightemitting diode," J. Appl. Phys. 106, 014508 (2009).

15. J. D. Joannopoulos, S. G. Johnson, J. N. Winn, and R. D. Meade, Photonic Crystals-Molding The Flow of Light, 2nd ed. (Princeton University Press, 2008), Chapter 5.

16. J. S. Lee, J. Lee, S. Kim, and H. Jeon, "Fabrication of reflective GaN mesa sidewalls for the application to high extraction efficiency LEDs," Phys. Stat. Sol. C 4, 2625-2628 (2007).

17. H. G. Kim, M. G. Na, H. K. Kim, H. Y. Kim, J. H. Ryu, T. V. Cuong, and C.-H. Hong, "Effect of periodic deflector embedded in InGaN/GaN light emitting diode," Appl. Phys. Lett. 90, 261117 (2007).

18. Y.-T. Moon, D.-J. Kim, J.-S. Park, J.-T. Oh, J.-M. Lee, and S.-J. Park, "Recovery of dry-etch induced surface damage on Mg-doped GaN by NH3 ambient thermal annealing," J. Vac. Sci. Technol. B 22, 489-491 (2004).

19. D. Zhuang and J. H. Edgar, "Wet etching of GaN, AlN, and SiC: A review," Mater. Sci. Eng. R 48, 1-46 (2005).

20. H. M. Ng, N. G. Weimann, and A. Chowdhury, "GaN nanotip pyramids formed by anisotropic etching," J. Appl. Phys. 94, 650 (2003). 